\title{
Smooth pointwise multipliers of modulation spaces
}

\author{
Ghassem Narimani
}

\begin{abstract}
Let $1<p, q<\infty$ and $s, r \in \mathbb{R}$. It is proved that any function in the amalgam space $W\left(H_{p^{\prime}}^{r}\left(\mathbb{R}^{d}\right), \ell_{\infty}\right)$, where $p^{\prime}$ is the conjugate exponent to $p$ and $H_{p^{\prime}}^{r}\left(\mathbb{R}^{d}\right)$ is the Bessel potential space, defines a bounded pointwise multiplication operator in the modulation space $M_{p, q}^{s}\left(\mathbb{R}^{d}\right)$, whenever $r>|s|+d$.
\end{abstract}

\section{Introduction}

By a pointwise multiplier from a function space $A$ into another function space $B$, we mean a function or distribution which defines a bounded linear mapping of $A$ into $B$ by pointwise multiplication. Pointwise multipliers arise in many different areas of mathematical analysis and its applications, for example coefficients of differential operators and symbols of more general pseudodifferential operators may be considered as pointwise multipliers. The theory of pointwise multipliers acting on several function spaces like Sobolev, Besov and TriebelLizorkin spaces have been developed by several mathematicians, among them are A. Devinatz, I.I. Hirschman, R. Strichartz, J. C. Polking, J. Peetre, V. Maz'ya, T. Shaposhnikova, and more recently by W. Sickel, T. Runst, J. Frank, and H. Koch. For the latest developments on pointwise multipliers of function spaces we refer to [11] which is entirely devoted to this topic.

Key Words: Pointwise multipliers, Modulation spaces, Amalgam spaces, Bessel potential spaces.

2010 Mathematics Subject Classification: 47B38, 42B35, 46 E35.

Received: February, 2011

Revised: March, 2011.

Accepted: February, 2012. 
In this paper we will consider the pointwise multipliers of modulation spaces $M_{p, q}^{s}\left(\mathbb{R}^{d}\right)$. (See section 2 for definitions). Modulation spaces are a family of smoothness spaces which are defined and used effectively by H. G. Feichtinger in early 1980s. They have origins in abstract and applied harmonic analysis. We refer to [4] and [6] for further historical and technical information about modulation spaces. Applications of modulation spaces in vast areas of mathematical and engineering problems, from signal analysis to the theory of differential and pseudodifferential operators, implies the necessity for a closer study of one of the most important operators on these spaces, namely, the multiplication operators.

One motivation for this work comes from a theorem about pointwise multipliers of Besov spaces due to H. Triebel which states that for $\varrho>\max \left\{s, \frac{d}{p}-s\right\}$ any function in the Zygmund space $\mathcal{C}^{\varrho}$ is a pointwise multiplier for the Besov space $B_{p, q}^{s}$. See section 2.8 in [17] for the precise statement and further results. Due to close similarity between definitions of modulation spaces and Besov spaces by Fourier analytical methods, we expect that modulation spaces might enjoy similar smooth multiplier property. The other motivation comes from a corollary in [2] which states that the space of all pointwise multipliers of the Sobolev space $H_{2}^{s}\left(\mathbb{R}^{d}\right)$ is equal to the amalgam space $W\left(H_{2}^{s}\left(\mathbb{R}^{d}\right), \ell_{\infty}\right)$ for $s>\frac{d}{2}$. By the fact that $H_{2}^{s}\left(\mathbb{R}^{d}\right)=M_{2,2}^{s}$, the question arises whether a similar result holds true for other modulation spaces $M_{p, q}^{s}$. We remark that aforementioned result on pointwise multipliers of the Sobolev spaces was known, before appearing in [2], and is due to Srichartz, cf. [15]. However, the idea of using the amalgam spaces for it's proof in [2] was inspiring to us.

In what follows we will give an answer to the questions raised above, more precisely, we prove that any function in the amalgam space $W\left(H_{p^{\prime}}^{r}\left(\mathbb{R}^{d}\right), \ell_{\infty}\right)$ is a pointwise multiplier from the modulation space $M_{p, q}^{s}\left(\mathbb{R}^{d}\right)$ into itself, where $1<p, q<\infty, s \in \mathbb{R}$, and $r>|s|+d$. Here $d$ is the dimension of the underlying Euclidean space $\mathbb{R}^{d}$.

We remark that we will not consider the theory of Fourier multipliers of modulation spaces. We just mention that the theory of Fourier multipliers of modulation spaces is considered in [5], where a characterization of Fourier multipliers of modulation spaces, by using amalgam spaces, is given.

\section{Preliminaries and Notations}

In this section we fix notations and provide necessary definitions.

General definitions. If $X$ and $Y$ are two quantities (typically nonnegative), we use $X \lesssim Y$ to denote the statement that $X \leq C Y$ for some absolute constant $C>0$. Also we use $X \sim Y$ to denote the statement $X \lesssim Y \lesssim X$. All function spaces in this paper are considered on the $d$ - 
dimensional Euclidean space $\mathbb{R}^{d}$. $\mathscr{S}$ and $\mathscr{S}^{\prime}$ denote the Schwartz space of rapidly decreasing smooth functions and it's dual space of tempered distributions, respectively, with their usual topologies. For two function spaces $X$ and $Y, X \hookrightarrow Y$ means that, $X$ is continuously embedded into $Y$. $\mathscr{F}$ denotes the Fourier transform normalized as

$$
\mathscr{F} f(y)=\int_{\mathbb{R}^{d}} f(x) e^{-2 \pi \mathrm{i} x \cdot y} d x
$$

which acts isomorphically and continuously on $\mathscr{S}$ and $\mathscr{S}^{\prime}$ and is a unitary operator on $L_{2}$. We will extensively use the weight functions

$$
w_{s}(y)=\langle y\rangle^{s}=\left(1+|y|^{2}\right)^{\frac{s}{2}}, \quad s \in \mathbb{R} .
$$

The operators of translation and modulation are defined by

$$
T_{y} f(x)=f(x-y) \quad \text { and } \quad M_{y} f(x)=e^{2 \pi \mathrm{i} x \cdot y} f(x),
$$

respectively.

Banach spaces of functions and distributions. For a Banach space $A$ and $f \in A$, the norm of $f$ in $A$ will be denoted by $\|f: A\|$ or $\|f\|_{A}$. For $1 \leq p \leq \infty$, $L_{p}$ is the Lebesgue space on $\mathbb{R}^{d}, \ell_{p}$ is the corresponding sequence space on $\mathbb{Z}^{d}$, and $\ell_{q}^{s}$ is the weighted sequence spaces with weight $w_{s}$. For $1 \leq p \leq \infty$ and $s \in \mathbb{R}$, the Bessel potential space is defined to be the set of all tempered distributions $f$ such that $\left\|f: H_{p}^{s}\right\|:=\left\|\mathscr{F}^{-1} w_{s} \mathscr{F} f: L_{p}\right\|<\infty$. With this norm $H_{p}^{s}$ is a Banach space. Now we define amalgam spaces which play a central role in the whole theory of modulation spaces. Amalgam spaces have been defined in more general settings in [1], which still preserves a lot of nice properties and includes as a special case the spaces defined below. Let $B$ be a Banach space of functions or tempered distributions such that $\mathscr{S} \hookrightarrow B$ and which is module over $\mathscr{S}$, i.e.

$$
\forall \phi \in \mathscr{S}, \forall f \in B, \quad \phi . f \in B .
$$

Let $\varphi$ be an infinitely differentiable function with compact support such that $\sum_{k \in \mathbb{Z}^{d}} T_{k} \varphi(x)=1$, for all $x \in \mathbb{R}^{d}$. Now letting $\varphi_{k}(x)=T_{k} \varphi(x)$, the amalgam space $W\left(B, \ell_{q}^{s}\right)$ is defined to be the set of all tempered distributions $f$ for which the norm defined by

$$
\left\|f: W\left(B, \ell_{q}^{s}\right)\right\|=\left\|\left\{\left\|\varphi_{k} f\right\|_{B}\right\}_{k \in \mathbb{Z}^{d}}: \ell_{q}^{s}\right\|
$$

is finite. $W\left(B, \ell_{q}^{s}\right)$ is a Banach space of tempered distributions. An example of a amalgam space which will be used is $W\left(H_{p}^{s}, \ell_{\infty}\right)$. Also we will use the notion of amalgam space in the definition of modulation spaces. Let $\mathscr{F} L_{p}$ 
denote the image of $L_{p}$ under the Fourier transform. Recall that the Fourier transform maps $\mathscr{S}^{\prime}$ into itself and $L_{p} \subseteq \mathscr{S}^{\prime}$. $\mathscr{F} L_{p}$ is a Banach space of tempered distributions with the norm defined by $\left\|f: \mathscr{F} L_{p}\right\|=\left\|\mathscr{F}^{-1} f: L_{p}\right\|$. Let $\varphi$ be as above. For $s \in \mathbb{R}^{d}$ and $1 \leq p, q \leq \infty$, the modulation space $M_{p, q}^{s}$ is the set of all tempered distributions $f$ for which the norm defined by

$$
\left\|f: M_{p, q}^{s}\right\|=\left\|\mathscr{F} f: W\left(\mathscr{F} L_{p}, \ell_{q}^{s}\right)\right\|=\left(\sum_{k \in \mathbb{Z}^{d}}\left\|\mathscr{F}^{-1} \varphi_{k} \mathscr{F} f\right\|_{p}^{q}\langle k\rangle^{s q}\right)^{\frac{1}{q}}
$$

is finite. For $s \in \mathbb{R}$ and $1 \leq p, q \leq \infty, M_{p, q}^{s}$ is a Banach space of tempered distributions. The family of modulation spaces contains some well-known function spaces, more precisely $M_{2,2}=L_{2}, M_{2,2}^{s}=H_{2}^{s}$, the Sobolev space and $M_{1,1}=S_{\circ}$, a well-known Banach algebra in abstract and applied harmonic analysis, called Feichtinger algebra. It is possible, and also more common in time-frequency analysis texts (see [6]), to define modulation spaces, equivalently, by using the notion of short time Fourier transform (STFT), but we found the definition given above more appropriate for our purpose. Modulation spaces are suitable spaces for many purposes in mathematical analysis and it's applications such as time-frequency analysis and PDE's. We have the following continuous embeddings between modulation spaces

$$
p_{1} \leq p_{2}, \quad q_{1} \leq q_{2}, \quad s_{1} \leq s_{2} \quad \Longrightarrow \quad M_{p_{1}, q_{1}}^{s_{1}} \hookrightarrow M_{p_{2}, q_{2}}^{s_{2}} .
$$

Also we have the following continuous embeddings between modulation ad Lebesgue spaces

$$
M_{p, q}^{s_{1}} \hookrightarrow L_{p} \hookrightarrow M_{p, q}^{s_{2}},
$$

for $s_{1}>\frac{d}{q^{\prime}}$ and $s_{2}<-\frac{d}{q}$, and

$$
M_{p, 1} \hookrightarrow L_{p} \hookrightarrow M_{p, \infty}
$$

For $1 \leq p, q<\infty$ and $s \in \mathbb{R}$, the dual space of $M_{p, q}^{s}$ is $M_{p^{\prime}, q^{\prime}}^{-s}$.

\section{Pointwise multipliers}

In the rest of this paper we will be concerned with the pointwise multipliers of modulation spaces. Let $A$ be a function space. $g \in \mathscr{S}^{\prime}$ is called a pointwise multiplier for the space $A$, if there exists $C>0$ such that

$$
\|g f: A\| \leq C\|f: A\|
$$

for all $f \in A$. In other words, $g$ is a pointwise multiplier if $f \mapsto g f$ defines a bounded mapping in $A$. The smallest $C$ satisfying 3.1 is called the multiplier 
norm of $g$ and is denoted by $\|g: M(A)\|$. Depending on the function space $A$, one might make clear that what is meant by the pointwise multiplication in 3.1, where $g$ or $f$ may be singular distributions. For a discussion of this problem see [10]. Here for arbitrary $f, g \in \mathscr{S}^{\prime}$, we define $f \cdot g$ as follows: For $n \in \mathbb{N}$, let $I_{n}=\left\{k \in \mathbb{Z}^{d}:|k| \leq n\right\}$. Then we define

$$
f \cdot g=\lim _{n \rightarrow \infty} \sum_{k, l \in I_{n}}\left[\mathscr{F}^{-1} \varphi_{k} \mathscr{F} f\right]\left[\mathscr{F}^{-1} \varphi_{l} \mathscr{F} f\right],
$$

whenever this limit exists in $\mathscr{S}^{\prime}$.

In general we will denote the space of all pointwise multipliers of a function space $A$ by $M(A)$. The following result on pointwise multipliers of Bessel potential spaces is due to Strichartz [15]. See also [11] for further results on multipliers of Bessel potential spaces.

Theorem 3.1. Let $1<p<\infty, s \in \mathbb{R}$ and $s>\frac{d}{p}$, then

$$
M\left(H_{p}^{s}\right)=W\left(H_{p}^{s}, \ell_{\infty}\right) .
$$

As we mentioned in the introduction we will not deal with Fourier multipliers of function spaces, however, to prove the main result we will need an estimate for a Fourier multiplier for $L_{p}$. We say that $m \in L_{\infty}$ is a Fourier multiplier for $L_{p}$ if

$$
\left\|\mathscr{F}^{-1} m \mathscr{F} f: L_{p}\right\| \leq C\left\|f: L_{p}\right\|
$$

for all $f \in \mathscr{S}$. The space of all Fourier multipliers of $L_{p}$ is denoted by $M_{\mathscr{F}}\left(L_{p}\right)$. The smallest constant $C$, such that 3.3 holds true, is denoted by $\left\|f: M_{\mathscr{F}}\left(L_{p}\right)\right\|$.

Lemma 3.2. Let $r \in \mathbb{R}, 1 \leq p \leq \infty$ and $k \in \mathbb{Z}$. Then

$$
\left\|w_{r} \varphi_{k}: M_{\mathscr{F}}\left(L_{p}\right)\right\| \lesssim w_{r}(k)
$$

Proof. Since $L_{1} * L_{p} \hookrightarrow L_{p}$, we have $\mathscr{F} L_{1} \cdot \mathscr{F} L_{p} \hookrightarrow \mathscr{F} L_{p}$ and hence $\mathscr{F} L_{1} \hookrightarrow$ $M\left(\mathscr{F}\left(L_{p}\right)\right)=M_{\mathscr{F}}\left(L_{p}\right)$ with $\left\|\cdot: M_{\mathscr{F}}\left(L_{p}\right)\right\| \lesssim\left\|\cdot: \mathscr{F} L_{1}\right\|$. Consequently, we 
have

$$
\begin{aligned}
\left\|w_{r} \varphi_{k}: M_{\mathscr{F}}\left(L_{p}\right)\right\| & \lesssim\left\|w_{r} \varphi_{k}: \mathscr{F}\left(L_{1}\right)\right\| \\
& =\int_{\mathbb{R}^{d}}\left|\mathscr{F}\left(w_{r} \varphi_{k}\right)(\xi)\right| d \xi \\
& =\int_{\mathbb{R}^{d}}\left|\mathscr{F}\left(w_{r} \varphi_{k}\right)(\xi)\right| w_{d+1}(\xi) w_{-d-1}(\xi) d \xi \\
& \lesssim\left\|\mathscr{F}\left(w_{r} \varphi_{k}\right) w_{d+1}: L_{\infty}\right\| \\
& \lesssim\left\|\mathscr{F}^{-1} w_{d+1} \mathscr{F}\left(w_{r} \varphi_{k}\right): L_{1}\right\| \\
& \lesssim \sum_{|\alpha| \leq d+1}\left\|D^{\alpha}\left(w_{r} \varphi_{k}\right): L_{1}\right\| \\
& \lesssim w_{r}(k) .
\end{aligned}
$$

Here $D$ is the differential operator, $\alpha=\left(\alpha_{1}, \cdots, \alpha_{d}\right)$ is a multi-index, $|\alpha|=$ $\alpha_{1}+\cdots+\alpha_{d}$ is it's length, and in inequality 3.4 we have used RiemannLebesgue's lemma.

Theorem 3.3. Let $s, r \in \mathbb{R}, r>|s|+d$ and $1<p<\infty$. Then

$$
W\left(H_{p^{\prime}}^{r}, \ell_{\infty}\right) \hookrightarrow M\left(M_{p, q}^{s}\right) .
$$

Proof. We proceed the proof in 3 steps.

Step 1. We have

$$
\begin{aligned}
\left\|f: M_{p, q}^{s}\right\| & =\left\|\left\{\left\|\mathscr{F}^{-1} \varphi_{k} \mathscr{F} f\right\|: L_{p}\right\}_{k \in \mathbb{Z}^{d}}: \ell_{q}^{s}\right\| \\
& =\left\|\left\{\left\|\mathscr{F}^{-1} T_{k} w_{r} T_{k} w_{-r} \varphi_{k} \mathscr{F} f: L_{p}\right\|\right\}_{k \in \mathbb{Z}^{d}}: \ell_{q}^{s}\right\| \\
& =\left\|\left\{\left\|\mathscr{F}^{-1} T_{k} w_{r} \varphi_{k} \mathscr{F} \mathscr{F}^{-1} T_{k} w_{-r} \mathscr{F} f\right\|: L_{p}\right\}_{k \in \mathbb{Z}^{d}}: \ell_{q}^{s}\right\| \\
& \leq\left\|\left\{\left\|T_{k} w_{r} \varphi_{k}: M_{\mathscr{F}}\left(L_{p}\right)\right\|\left\|\mathscr{F}^{-1} T_{k} w_{-r} \mathscr{F} f: L_{p}\right\|\right\}_{k \in \mathbb{Z}^{d}}: \ell_{q}^{s}\right\| \\
& \leq\left\|w_{r} \varphi: M_{\mathscr{F}}\left(L_{p}\right)\right\|\left\|\left\{\left\|\mathscr{F}{ }^{-1} w_{-r} \mathscr{F} M_{-k} f: L_{p}\right\|\right\}_{k \in \mathbb{Z}^{d}}: \ell_{q}^{s}\right\| \\
& \lesssim\left\|\left\{\left\|M_{-k} f: H_{p}^{-r}\right\|\right\}: \ell_{q}^{s}\right\| .
\end{aligned}
$$

Step 2. For $j \in \mathbb{Z}^{d}$ let

$$
j^{*}=\left\{i \in \mathbb{Z}^{d}: \operatorname{supp}\left(\varphi_{i}\right) \cap \operatorname{supp}\left(\varphi_{j}\right) \neq \emptyset\right\} .
$$

Since

$$
\begin{aligned}
\left\|M_{-k} f: H_{p}^{-r}\right\| & =\left\|\mathscr{F}^{-1} w_{-r} \mathscr{F}\left(M_{-k} f\right): L_{p}\right\| \\
& =\left\|\mathscr{F}^{-1}\left(T_{k} w_{-r}\right) \mathscr{F} f: L_{p}\right\|
\end{aligned}
$$


and

$$
\begin{aligned}
\mathscr{F}^{-1}\left(T_{k} w_{-r}\right) \mathscr{F} f & =\sum_{j \in \mathbb{Z}^{d}} \sum_{i \in j^{*}} \mathscr{F}^{-1}\left(T_{k} w_{-r}\right) \varphi_{j} \varphi_{i} \mathscr{F} f \\
& =\sum_{j \in \mathbb{Z}^{d}} \sum_{i \in j^{*}} \mathscr{F}^{-1} \varphi_{i}\left(T_{k} w_{-r}\right) \mathscr{F} \mathscr{F}^{-1} \varphi_{j} \mathscr{F} f
\end{aligned}
$$

with convergence in $\mathscr{S}^{\prime}$, we have

$$
\left\|M_{-k} f: H_{p}^{-r}\right\| \leq \sum_{j \in \mathbb{Z}^{d}} \sum_{i \in j^{*}}\left\|\varphi_{i}\left(T_{k} w_{-r}\right): M_{\mathscr{F}}\left(L_{p}\right)\right\|\left\|\mathscr{F}^{-1} \varphi_{j} \mathscr{F} f: L_{p}\right\| .
$$

Now by Lemma 3.2

$$
\left\|\varphi_{i} T_{k} w_{-r}: M_{\mathscr{F}}\left(L_{p}\right)\right\| \lesssim w_{-r}(k-i)
$$

and therefore

$$
\left\|M_{-k} f: H_{p}^{-r}\right\| \lesssim \sum_{j \in \mathbb{Z}^{d}} \sum_{i \in j^{*}} w_{-r}(k-i)\left\|\mathscr{F}^{-1} \varphi_{j} \mathscr{F} f: L_{p}\right\| .
$$

Since $i \in j^{*}$, we have $w_{-r}(k-i) \sim w_{-r}(k-j)$, and therefore we may replace $i$ by $j$ in 3.6. After this substitution, by using the fact $\left|j^{*}\right| \sim 1$, we see that the right hand side of 3.6 is actually a convolution of two sequences $\left\{w_{-r}(j)\right\}_{j \in \mathbb{Z}^{d}}$ and $\left\{\left\|\mathscr{F}_{-1} \varphi_{j} \mathscr{F} f: L_{p}\right\|\right\}_{j \in \mathbb{Z}^{d}}$. By invoking Young's inequality and using the $w_{|s|}$-moderateness of the weight function $w_{s}$, i.e.

$$
w_{s}(x+y) \leq w_{|s|}(x) w_{s}(y),
$$

we have

$$
\left\|\left\{\left\|M_{-k} f: H_{p}^{-r}\right\|\right\}_{k \in \mathbb{Z}^{d}}: \ell_{q}^{s}\right\| \lesssim\left\|\left\{w_{-r}(j)\right\}_{j \in \mathbb{Z}^{d}}: \ell_{1}^{|s|}\right\|\left\|\left\{\left\|\mathscr{F}^{-1} \varphi_{j} \mathscr{F} f: L_{p}\right\|\right\}_{j \in \mathbb{Z}^{d}}: \ell_{q}^{s}\right\| .
$$

Since $r>|s|+d$, we have $\left\|\left\{w_{-r}(j)\right\}_{j \in \mathbb{Z}^{d}}: \ell_{1}^{|s|}\right\|<\infty$, and finally we arrive at

$$
\left\|\left\{\left\|M_{-k} f: H_{p}^{-r}\right\|\right\}_{k \in \mathbb{Z}^{d}}: \ell_{q}^{s}\right\| \lesssim\left\|f: M_{p, q}^{s}\right\| .
$$

Step 3. Since the operator of multiplication is self-adjoint we have $M\left(H_{p}^{-r}\right)=$ $M\left(H_{p^{\prime}}^{r}\right)$, with equality of the corresponding norms. Finally, Theorem 3.1 together with 3.5 and 3.7 completes the proof.

By [5, Theorem 15] the space of pointwise multipliers of $W\left(\mathscr{F} L_{p}, \ell_{q}\right)$ can be characterized as the amalgam space $W\left(M_{\mathscr{F}}\left(L_{p}\right), \ell_{\infty}\right)$, with equivalent norms. On the other hand $M_{p, p}=W\left(\mathscr{F} L_{p}, \ell_{p}\right)$, with equal norms . Therefore we have the following characterization theorem for pointwise multipliers of modulation spaces $M_{p, p}$. 
Theorem 3.4. Let $1 \leq p \leq \infty$. Then

$$
M\left(M_{p, p}\right)=W\left(M_{\mathscr{F}}\left(L_{p}\right), \ell_{\infty}\right),
$$

and specifically,

$$
M\left(M_{1,1}\right)=M\left(M_{\infty, \infty}\right)=W\left(\mathscr{F} L_{1}, \ell_{\infty}\right) .
$$

Note that the last equality in 3.8 follows from

$$
M_{\mathscr{F}}\left(L_{1}\right)=\mathscr{F} \mathbf{M},
$$

where $\mathbf{M}$ is the space of bounded regular Borel measures on $\mathbb{R}^{d}$, and the fact that $(\mathscr{F} \mathbf{M})_{l o c}=\left(\mathscr{F} L_{1}\right)_{l o c}$.

In the rest we will compare Theorem 3.3 with a known result in the literature concerning the pointwise multipliers of modulation spaces. The following theorem on convolution of amalgam spaces is due to Feichtinger. See [1] for a general version of this theorem.

Theorem 3.5. Let $1 \leq p, q, p_{1}, q_{1}, p_{2}, q_{2} \leq \infty$ such that

$$
\frac{1}{p_{2}}=\frac{1}{p}+\frac{1}{p_{1}} \quad \text { and } \quad \frac{1}{q_{2}}+1=\frac{1}{q}+\frac{1}{q_{1}},
$$

then

$$
W\left(\mathscr{F} L_{p}, \ell_{q}\right) * W\left(\mathscr{F} L_{p_{1}}, \ell_{q_{1}}\right) \hookrightarrow W\left(\mathscr{F} L_{p_{2}}, \ell_{q_{2}}\right)
$$

Since modulation spaces are, by definition, images of amalgam spaces under inverse Fourier transform, 3.10 implies that

$$
M_{p, q} \cdot M_{p_{1}, q_{1}} \hookrightarrow M_{p, q},
$$

which in terms of pointwise multipliers means that

$$
M_{p, q} \hookrightarrow M\left(M_{p_{1}, q_{1}}, M_{p_{2}, q_{2}}\right) .
$$

Here $M\left(M_{p_{1}, q_{1}}, M_{p_{2}, q_{2}}\right)$ denotes the space of all pointwise multipliers from $M_{p_{1}, q_{1}}$ to $M_{p_{2}, q_{2}}$. In the special case when $p_{1}=p_{2}$ and $q_{1}=q_{2}$ the embedding 3.12 turns to the following

$$
M_{\infty, 1} \hookrightarrow M\left(M_{p, q}\right) .
$$

The modulation space $M_{\infty, 1}$, called Sjöstrand algebra, is a Banach algebra of continuous functions with pointwise product. It provides a symbol class for an algebra of bounded pseudodifferential operators on $L_{2}$ (and also on all modulation spaces) which has the spectral invariance property. See [14] and [7] for properties and applications of $M_{\infty, 1}$ in the theory of pseudodifferential operators. To see how much $W\left(H_{p}^{s}, \ell_{\infty}\right)$ and $M_{\infty, 1}$ are closed to each other, at least set theoretically, we prove the following embeddings. 
Proposition 3.6. For $1 \leq p \leq \infty$ and $s>d$,

(i) $H_{p}^{s} \hookrightarrow M_{\infty, 1} \hookrightarrow W\left(\mathscr{F} L_{1}, \ell_{\infty}\right)$

(ii) $H_{p}^{s} \hookrightarrow W\left(H_{p}^{s}, \ell_{\infty}\right) \hookrightarrow W\left(\mathscr{F} L_{1}, \ell_{\infty}\right)$

Proof. (i) Let $B_{p, q}^{s}$ and $F_{p, q}^{s}$ denote the Besov and Triebel-Lizorkin space, respectively. Then $H_{p}^{s}=F_{p, 2}^{s}$ (see [17]). By Proposition 2.3.2 in [17] we have the following sequence of embeddings

$$
F_{p, 2}^{s} \hookrightarrow B_{p, \max \{p, 2\}}^{s} \hookrightarrow B_{p, 1}^{d} .
$$

We need to show that

$$
B_{p, 1}^{d} \hookrightarrow M_{\infty, 1} .
$$

To this end, let $\left\{\psi_{n}\right\}_{n \in \mathbb{N}^{\circ}}$ be the dyadic partition of unity used in the definition of Besov spaces. Let $N_{n}=\left\{k \in \mathbb{Z}^{d}: \operatorname{supp} \varphi_{k} \cap \operatorname{supp} \psi_{n} \neq \emptyset\right\}$. Then

$$
\begin{aligned}
\left\|f: M_{\infty, 1}\right\| & =\sum_{k \in \mathbb{Z}^{d}}\left\|\mathscr{F}^{-1} \varphi_{k} \mathscr{F} f\right\|_{\infty} \\
& =\sum_{k \in \mathbb{Z}^{d}}\left\|\sum_{n \in \mathbb{N}_{\circ}} \mathscr{F}^{-1} \psi_{n} \varphi_{k} \mathscr{F} f\right\|_{\infty} \\
& \leq \sum_{n \in \mathbb{N}_{\circ}} \sum_{k \in N_{n}}\left\|\mathscr{F}^{-1} \varphi_{k} * \mathscr{F}^{-1} \psi_{n} \mathscr{F} f\right\|_{\infty} \\
& \lesssim \sum_{n \in \mathbb{N}_{\circ}} \sum_{k \in N_{n}}\left\|\mathscr{F}^{-1} \varphi_{k}\right\|_{p^{\prime}}\left\|\mathscr{F}^{-1} \psi_{n} \mathscr{F} f\right\|_{p} \\
& \lesssim \sum_{n \in \mathbb{N}_{\circ}} 2^{n d}\left\|\mathscr{F}^{-1} \psi_{n} \mathscr{F} f\right\|_{p}=\left\|f: B_{p, 1}^{d}\right\|
\end{aligned}
$$

The second embedding follows from embeddings of amalgam spaces as in $[3$, Theorem 9].

(ii) For $k \in \mathbb{Z}^{d}, T_{k}$ is an isometry on $W\left(H_{p}^{s}, \ell_{\infty}\right)$ and $\varphi \in \mathscr{S}$ is a pointwise multiplier for $H_{p}^{s}$. Therefore

$$
H_{p}^{s} \hookrightarrow W\left(H_{p}^{s}, \ell_{\infty}\right)
$$

(This embedding can also be deduced from the localization property of $H_{p}^{s}$, see [15].) We prove the second embedding. By [13, Lemma 1], the modulation space $M_{\infty, 1}$ is locally the same as $\mathscr{F} L_{1}$, i.e. $\left(M_{\infty, 1}\right)_{l o c}=\left(\mathscr{F} L_{1}\right)_{l o c}$. Therefore $W\left(\mathscr{F} L_{1}, \ell_{\infty}\right)=W\left(M_{\infty, 1}, \ell_{\infty}\right)$. Now (i) and the embedding properties of amalgam spaces proved in [1] will give the result. 
Remark 3.7. The embedding 3.14 used in the above proof is new and it is not comparable with existing embeddings of modulation and Besov spaces in [16] and [12]. It is stronger than the embedding of Heil, Ramanathan and Topiwala in [9] and [8], which states that $B_{\infty, \infty}^{\varrho}=C^{\varrho} \hookrightarrow M_{\infty, 1}$, whenever $\varrho>d$.

\section{References}

[1] H. G. Feichtinger, Banach spaces of distributions of Wiener's type and interpolation. Functional analysis and approximation (Oberwolfach, 1980), pp. 153-165, Internat. Ser. Numer. Math., 60, Birkhäuser, Basel-Boston, Mass., 1981.

[2] H. G. Feichtinger, Modulation spaces on locally compact Abelian groups, in Proc. Internat. Conf. on Wavelets and Applications (Chennai, 2002), R. Radha, M. Krishna, S. Thangavelu (eds.), New Delhi Allied Publishers, 2003, p.1-56. (Reprint of the 1983 technical report.)

[3] H. G. Feichtinger, Generalized amalgams, with applications to Fourier transform, Canad. J. Math. 42(3) (1990), 395-409.

[4] H. G. Feichtinger, Modulation Spaces: Looking Back and Ahead, Sampl. Theory Signal Image Process., 5(2) (2006), 109-140.

[5] H. G. Feichtinger and G. Narimani, Fourier multipliers of classical modulation spaces, Appl. Comput. Harmon. Anal. 21 (2006), 349-359.

[6] K. Gröchenig, Foundations of time-frequency analysis, Applied and Numerical Harmonic Analysis. Birkhäuser Boston, Inc., Boston, MA, 2001.

[7] K. Gröchenig, Time-frequency analysis of Sjöstrands class, Rev. Mat. Iberoamericana, 22 (2006), 703-724.

[8] K. Gröchenig and C. Heil, Modulation spaces and pseudodifferential operators, Integr. equ. oper. theory, 34(4) (1999), 439-457.

[9] C. Heil, J. Ramanathan and P. Topiwala, Singular values of compact pseudodifferential operators, J. Funct. Anal., 150 (1997), 426-452.

[10] H. Koch and W. Sickel, Pointwise multipliers of Besov spaces of smoothness zero and spaces of continuous functions, Rev. Mat. Iberoamericana, 18 (2002), 587-626.

[11] V. G. Maz'ya and T. O. Shaposhnikova, Theory of Sobolev multipliers. With applications to differential and integral operators, Grundlehren der Mathematischen Wissenschaften, 337. Springer-Verlag, Berlin, 2009. 
[12] K. A. Okoudjou, Embeddings of some classical Banach spaces into modulation spaces, Proc. Amer. Math. Soc., 132 (2004), 1639-1647.

[13] K. A. Okoudjou, A Beurling-Helson type theorem for modulation spaces, J. Funct. Spaces Appl., 7(1) (2009), 33-41.

[14] J. Sjöstrand, An algebra of pseudodifferential operators, Math. Res. Lett. 1(2) (1994), 185-192.

[15] R. S. Strichartz, Multipliers on fractional Sobolev spaces, J. Math. and Mech., 16(9) (1967), 1031-1060.

[16] J. Toft, Continuity properties for modulation spaces, with applications to pseudo-differential calculus-I, J. Funct. Anal., 207 (2004), 399-429.

[17] H. Triebel, Theory of function spaces. Monographs in Mathematics, 78. Birkhäuser Verlag, Basel, 1983.

[18] H. Triebel, Theory of function spaces II, Monographs in Mathematics, 84. Birkhäuser Verlag, Basel, 1992.

Ghassem NARIMANI,

Department of Mathematics and Applications,

University of Mohaghegh Ardabili,

Ardabil 56199-11367, Iran.

Email: ghnarimani@gmail.com 
